\title{
OILSEED RAPE PLANTING AREA EXTRACTION BY SUPPORT VECTOR MACHINE USING LANDSAT TM DATA
}

\author{
Yuan Wang ${ }^{1,2}$, Jingfeng Huang ${ }^{1,3}$, Xiuzhen Wang ${ }^{4}$, Fumin Wang ${ }^{1,3}$, \\ Zhanyu Liu ${ }^{1,3}$, Junfeng $\mathrm{Xu}^{1,3^{*}}$ \\ ${ }^{1}$ Institute of Agricultural Remote Sensing and Information Technology, Zhejiang University, \\ 310029, Hangzhou, P. R. China \\ ${ }^{2}$ Ministry of Education Key Laboratory of Environmental Remediation and Ecological \\ Health, Zhejiang University, 310029, Hangzhou, P. R. China \\ ${ }^{3}$ Key Laboratory of Agricultural Remote Sensing and Information System, Zhejiang Province \\ ${ }^{4}$ Zhejiang Meteorological Institute, 310004, Hangzhou P. R. China \\ * Ccorrespondence Author, Address: Institute of Agricultural Remote Sensing and \\ Information Technology, Zhejiang University, 310029, Hangzhou, P. R. China, Tel: \\ +86(0)5718697 1830, Fax:86-571-86971831,E-mail: xjf11@zju.edu.cn
}

Abstract: One parametric classify (Maximum likelihood classify, MLC for short) and two non-parametric classifiers (Adaptive resonance theory mappings and Support vector machines, ARTMAP and SVM for short) were presented in this study. Base on the confusion matrix and the pixels fuzzy analysis, the nonparametric classifier may be a more preferable approach than the parametric classifier for some remote sensing applications and deserves further investigation. The ARTMAP classify represent much best than the rest of classify, especially for the grade of pure pixel (90-100\% pureness), Kappa coefficients and overall accuracy were nearly $100 \%$. The higher pureness the pixels were, the better classification accuracy was got.

Keywords: planting area extraction, SVM, ARTMAP, neural networks, oilseed rape, confusion matrix

Please use the following format when citing this chapter:

Wang, Y., Huang, J., Wang, X., Wang, F., Liu, Z. and Xu, J., 2009, in IFIP International Federation for Information Processing, Volume 294, Computer and Computing Technologies in Agriculture II, Volume 2, eds. D. Li, Z. Chunjiang, (Boston: Springer), pp. 861-870. 


\section{INTRODUCTION}

Spectral classifiers use only the spectral information of the pixel to be classified. Spectral classifiers are split into two main categories: (a) parametric classifiers, if they assume the existence of an underlying probability distribution of the data, and $(b)$ non-parametric classifiers, if they do not assume anything about the probability distribution. When classifying a multi-spectral image by using a spectral classifier such as the classical maximum likelihood classifier(MLC), the resulting thematic map will usually give the overall impression of a 'noisy' classification. The thematic map that they obtain is composed by many isolated labeled pixels inside patches of other classes giving the overall impression of a noisy classification. This negative effect is more evident when there is overlapping between the training sets in the spectral space (Cortijo et al., 1995). When this occurs the traditional quadratic ML classifier and the linear classifiers are not able to obtain a high accuracy classification due to the fixed-form decision boundaries they impose.

In most classification applications the assumption that the forms of the underlying density functions are known is unrealistic. The common parametric forms rarely fit the densities actually encountered in practice. For instance, the parametric models manage unimodal densities, whereas many practical problems present multi-modal densities (Duda and Hart, 1973). The only available information is the training set and the classification rules must be built only from it with no additional assumptions.

In this study, One popular used parametric classify, Maximum likelihood classify (MLC), andtwo non-parametric classifiers were selected, support vector machine (SVM) (Cristianini and Shawe-Taylor, 2000) and ARTMAP (Vapnik, 1995) neural network. We shall show that alternative spectral classifiers may improve the accuracy of the classifications as they are able to define a wider set of decision boundaries between the clusters in the representation space, thus reducing the classification error rate. In this work, we perform a comparative study of different spectral classifiers, when the available training samples are highly overlapping in the representation space.

\section{MATERIALS AND METHODS}

\subsection{Study area and data acquisition}

An area of $5 \mathrm{~km} \times 5 \mathrm{~km}$ located around Haiyan country within the Zhejiang province, China was selected. Mapping urban and sub-urban land covers is 
also recognized as an important but difficult task (Barnsley and Barr, 1996). In order to obtain the ground reference data, a precision tracking of the study area feature was got using a viliami grade GPS Receiver (Trimble GeoXT) in April to May, 2004, and created the detail feature vector map of the study area (Fig. 1). There were a variety of urban thematic and topographic features at the site. The main crops of this season were rape and wheat. The other crops were sporadic, blend in a mixed type. According to the area and attributes of the study area, the district features of the ground vector map category into residential area, rape, wheat, road, tree, water, grass and the other crops. Then transferred the features map to 1-meter, 3-meter and 30meter grid data. 1-meter grid data use for remote sensing images for simulation, 3-meter data for the pixel fuzzy analysis, got the pixel percentage of the 30-meterdata. 30-meter grid data as a ground reference data of TM image.

Landsat 5 TM data of the site were used. All analyses were based on the data acquired in six of the TM's six wavebands. These were TM bands 1, 2, 3, 4, 5 and 7. The Landsat 5 TM data was acquired in April 5th, 2004; correspond to the ground reference data, and can be safely assumed free of significant temporal differences in land cover. For the purpose of this study, the following five classes appropriate to the scene defined: residential area (built-up land), rape, wheat, road (road land including road, bridge, barren land and sand road), tree (including fruit trees and mulberry), water (water bodies, including lakes ponds, and channel), grass (including grassland, grass road, bush, vegetable and wasteland) and the other crops (other crops, including soybean, horsebean, leek, cotton and corn).

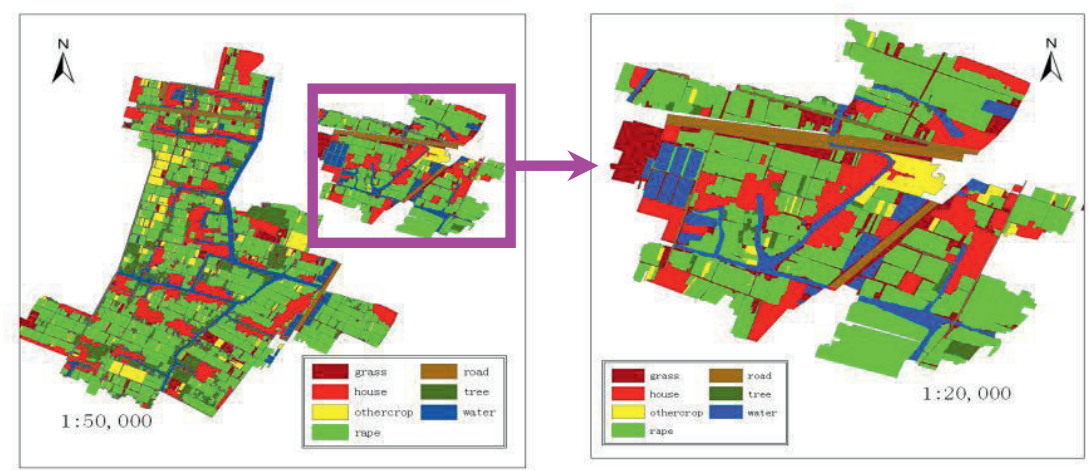

Fig.1: Sketch map of the study areas 


\subsection{Derivation of fuzzy ground data}

Mixed pixel problem has a great influence for remote sensing image classification accuracy. In order to analysis the impact of mixed-pixel, pixel purity data should be got. This study used 3-meter grid data, degrade to 30meter grid data, so have 1003 -meter lattices in one 30meter lattice. Calculating 3-meter lattice numbers of every ground features in the 30-meter lattice, got the percentage of every features in the pixels, expressed as the pixel features purity. Finally, the fuzzy ground data derived from the pixel features purity were stored as an eight-band image, one for each class.

Data handling and analysis were made in ENVI 4.2, ERDAS IMAGINE 8.7, ARCGIS 9.0, MATLAB 7.0 (Mathworks, USA), and LIBSVM 2.8.3 (Chang and Lin, 2001).

\section{RESULTS}

\subsection{Pixel Fuzzy Analysis}

In order to have a summary recognition of the study area, there was a brief statistic shown in Table 1.Among all 8 classes of the study area, grass is the largest class which have $33.68 \%$ of the area, followed by rape, tree, wheat, water, residential area, road and other crop, rape and wheat are the mainly crop of the area. Grass has the most parcels counts, the average area was only $1513.33 \mathrm{~m}^{2}$, which is only more than one pixels in TM image. The largest average area of the classes was tree, which was only same area as two pixels in TM image. So it is reasonable that the TM image of the study has a large number of mixture pixels. Based on the GPS ground data, the fuzzy analysis of the $30 \mathrm{~m}$ spatial resolution data was given followed.

Table 1. Average area and compactness of parcels in the study area

\begin{tabular}{ccccccccc}
\hline Class & Residential area & Rape & Wheat & Road & Tree & Water & Grass & Other crop \\
\hline $\begin{array}{c}\text { Count } \\
\text { Summation } \\
\left(\mathrm{m}^{2}\right)\end{array}$ & 125 & 894 & 151 & 117 & 501 & 132 & 1020 & 85 \\
$\begin{array}{c}\text { Average } \\
\left(\mathrm{m}^{2}\right)\end{array}$ & 1326.37 & 1499.86 & 1290.59 & 1196.77 & 1738.06 & 1475.9 & 1513.33 & 1550.42 \\
$\begin{array}{c}\text { Percentage } \\
(\%)\end{array}$ & 3.62 & 29.26 & 4.25 & 3.06 & 19.00 & 4.25 & 33.68 & 2.88 \\
$\begin{array}{c}\text { Standard } \\
\text { deviation }\end{array}$ & 883.39 & 870.39 & 846.41 & 935.35 & 857.19 & 870.75 & 863.10 & 822.59 \\
Variance & 780381.10 & 757582.01 & 716411.37 & 874887.51 & 734782.58 & 758213.56 & 744953.07 & 676654.36 \\
\hline
\end{tabular}


The fuzziness of TM image can be calculated from the proportion of one class in overall pixels that contain this class in the $30 \mathrm{~m}$ ground reference data. 7 grades was ranked in this study, $100 \%, 90-99 \%, 80-89 \%, 60-79 \%$, $40-59 \%, 20-39 \%$ and $1-19 \%$, the result shown in Table 2 . It was clear showed that in the pixels that contain class rape, the pure pixels only have $15.86 \%$, the pixels contain $1-19 \%$ of rape have $18.02 \%$ overall, $11.51 \%$, $11.35 \%, 15.54 \%, 13.87 \%$ and $13.87 \%$ for the $90-99 \%, 80-89 \%, 60-79 \%$, $40-59 \%$ and $20-39 \%$ pixels respectively. The residential area has $17.27 \%$ pure pixels, which was the most in all class, the least class was grass, and only have $1.53 \%$. Otherwise, the road and grass have $66.70 \%$ and $65.13 \%$ of $1-19 \%$ pixels.

Table 2. Percent of one object in pixels contained the object in reference map with $30 \mathrm{~m}$ resolution

\begin{tabular}{ccccccccc}
\hline Grade & Residential area & Rape & Wheat & Road & Tree & Water & Grass & Other crop \\
\hline $100 \%$ & 17.27 & 15.86 & 3.66 & 2.75 & 4.23 & 3.59 & 1.53 & 12.22 \\
$90-99 \%$ & 9.35 & 11.51 & 2.48 & 2.66 & 2.96 & 6.34 & 1.02 & 4.52 \\
$80-89 \%$ & 6.06 & 11.35 & 4.05 & 3.20 & 4.12 & 4.79 & 0.82 & 4.75 \\
$60-79 \%$ & 12.36 & 15.54 & 8.62 & 3.37 & 8.88 & 12.68 & 2.61 & 8.82 \\
$40-59 \%$ & 13.65 & 13.87 & 14.23 & 4.88 & 14.23 & 17.76 & 6.73 & 10.18 \\
$20-39 \%$ & 16.22 & 13.87 & 26.89 & 16.43 & 23.48 & 19.80 & 21.97 & 16.74 \\
$1-19 \%$ & 25.10 & 18.02 & 40.08 & 66.70 & 42.09 & 35.02 & 65.31 & 42.76 \\
Total & 100 & 100 & 100 & 100 & 100 & 100 & 100 & 100 \\
\hline
\end{tabular}

\subsection{Classification evaluation}

Because of the few pure pixels in TM image, the train data of the three classifies used the pixels which was more than $90 \%$ purity of all pixels, the total of train data was 2465 , the number of train data for residential area, rape, wheat, road, tree, water, grass and the other crops was 558, 1358, 47, $61,136,141,90$ and 74 respectively. The inputs of the classifies were the six wavebands of the TM image(bands 1,2,3,4,5 and 7), and the out put of the classifies was the code of each classes $(1,2,3,4,5,6,7$ and 8 for residential area, rape, wheat, road, tree, water, grass and the other crops, respectively). Maximum likelihood classifies (MLC) was automatic run in software ENVI. The SVM classify selected RBF kernel, and the train epoch was 10000 . In the ARTMAP classify, three alertness index was selected for train, 0.10 , 0.75 and 0.90 , finally used 0.75 as the alertness index because of the fine classification result.

The accuracy of each classification method is assessed based on its confusion matrix. Recall that the entry of $i$ th row and $j$ th column of this matrix is the number of sample pixels from the $j$ th class that have classified as belonging to the $i$ th class. Various indicators are derived from this matrix, 
such as overall accuracy, producer's accuracy, user's accuracy and Kappa coefficients. The confusion matrixes of each classification were showed in Table 3-5. The overall accuracy and Kappa coefficients denoted that ARTMAP has the highest value among the three classifies, followed by SVM, MLC has the lowest. Compared producer's accuracy and user's accuracy of different classifies; results showed that ARTMAP was not always the highest. The highest user's accuracy of wheat, road, tree and grass were ARTMAP, residential area and rape for MLC, water and other crop for SVM. The highest producer's accuracy of residential area, water, grass and other crop were ARTMAP, wheat, road and tree for MLC, rape for SVM. It is obvious that different classifies has differentia for different classes.

Table 3. The confusion matrix of classification by MLC

\begin{tabular}{ccccccccccc}
\hline \multirow{2}{*}{ Class } & \multicolumn{10}{c}{ Ground reference data } \\
\cline { 2 - 11 } & Residential area & Rape & Wheat & Road & Tree & Water & Grass Other crop & Total & User accuracy \\
\hline Residential area & 440 & 136 & 7 & 13 & 75 & 85 & 39 & 13 & 808 & 54.46 \\
Rape & 175 & 2150 & 60 & 13 & 129 & 62 & 117 & 52 & 2758 & 77.96 \\
Wheat & 17 & 125 & 115 & 0 & 13 & 12 & 23 & 2 & 307 & 37.46 \\
Road & 71 & 102 & 2 & 63 & 19 & 40 & 27 & 7 & 331 & 19.03 \\
Tree & 197 & 234 & 12 & 7 & 250 & 87 & 67 & 20 & 874 & 28.60 \\
Water & 57 & 88 & 2 & 15 & 36 & 233 & 33 & 6 & 470 & 49.57 \\
Grass & 101 & 254 & 29 & 11 & 71 & 29 & 68 & 15 & 578 & 11.76 \\
Other crop & 131 & 179 & 5 & 13 & 28 & 49 & 21 & 60 & 486 & 12.35 \\
Total & 1189 & 3268 & 232 & 135 & 621 & 597 & 395 & 175 & 6612 & 3379 \\
Prod.accuracy & 37.01 & 65.79 & 49.57 & 46.67 & 40.26 & 39.03 & 17.22 & 21.67 & $34.21 *$ & $51.10^{* *}$ \\
\hline
\end{tabular}

* Kappa coefficients, ** overall accuracy

Table 4. The confusion matrix of classification by SVM

\begin{tabular}{ccccccccccc}
\hline \multirow{2}{*}{ Class } & \multicolumn{10}{c}{ Ground reference data } \\
\cline { 2 - 11 } & Residential area Rape & Wheat & Road & Tree & Water & Grass & Other crop & Total User Accuracy \\
\hline Residential area & 760 & 319 & 15 & 71 & 200 & 224 & 95 & 49 & 1733 & 43.85 \\
Rape & 378 & 2818 & 155 & 80 & 265 & 157 & 243 & 98 & 4194 & 67.19 \\
Wheat & 0 & 28 & 58 & 0 & 1 & 1 & 3 & 0 & 91 & 63.74 \\
Road & 1 & 6 & 0 & 14 & 2 & 0 & 5 & 0 & 28 & 50.00 \\
Tree & 29 & 52 & 1 & 4 & 134 & 21 & 21 & 6 & 268 & 50.00 \\
Water & 19 & 36 & 1 & 10 & 13 & 189 & 19 & 2 & 289 & 65.40 \\
Grass & 2 & 7 & 2 & 7 & 2 & 1 & 3 & 1 & 25 & 12.00 \\
Other crop & 6 & 6 & 0 & 4 & 4 & 5 & 2 & 21 & 48 & 43.75 \\
Total & 1195 & 3272 & 232 & 190 & 621 & 598 & 391 & 177 & 6676 & 3997 \\
Prod.accuracy & 63.60 & 86.12 & 25.00 & 7.37 & 21.58 & 31.61 & 0.77 & 11.86 & $36.99 *$ & $59.87 * *$ \\
\hline
\end{tabular}


Table 5. The confusion matrix of classification by ARTMAP

\begin{tabular}{cccccccccccc}
\hline \multirow{2}{*}{ Class } & \multicolumn{10}{c}{ Ground reference data } \\
\cline { 2 - 12 } & Residential area & Rape & Wheat & Road & Tree & Water & Grass & Other crop & Total & User accuracy \\
\hline Residential area & 817 & 241 & 15 & 43 & 134 & 152 & 84 & 23 & 1509 & 54.14 \\
Rape & 246 & 2727 & 108 & 67 & 197 & 128 & 141 & 55 & 3669 & 74.33 \\
Wheat & 3 & 24 & 79 & 0 & 2 & 3 & 4 & 0 & 115 & 68.70 \\
Road & 6 & 12 & 1 & 43 & 1 & 5 & 5 & 0 & 73 & 58.90 \\
Tree & 39 & 75 & 6 & 5 & 211 & 24 & 18 & 5 & 383 & 55.09 \\
Water & 38 & 85 & 3 & 13 & 38 & 257 & 31 & 7 & 472 & 54.45 \\
Grass & 22 & 59 & 18 & 7 & 27 & 11 & 102 & 5 & 251 & 40.64 \\
Other crop & 22 & 44 & 2 & 12 & 11 & 18 & 5 & 81 & 195 & 41.54 \\
Total & 1193 & 3267 & 232 & 190 & 621 & 598 & 390 & 176 & 6667 & 4317 \\
Prod.accuracy & 68.48 & 83.47 & 34.05 & 22.63 & 33.98 & 42.98 & 26.15 & 46.02 & $47.63^{*}$ & $64.75^{* *}$ \\
\hline
\end{tabular}

* Kappa coefficients, ** overall accuracy

The overall accuracy and Kappa coefficients description previous only evaluated the total accuracy of classification; producer's accuracy and user's accuracy were discrete and always disagree with each other. In order to get much better classification evaluation, a new Classification evaluation index- "precision product" was used in this study, in was the product of producer's accuracy and user's accuracy, the higher of precision product of one class, the higher classification accuracy of this class. Fig. 2 showed the precision product of various classifiers. Rape has the highest classification accuracy for each classifies, which followed by residential area, water, wheat, tree, other crop and road, grass has the lowest classification accuracy. In the three classifies, the ARTMAP has the highest classification accuracy for each class.

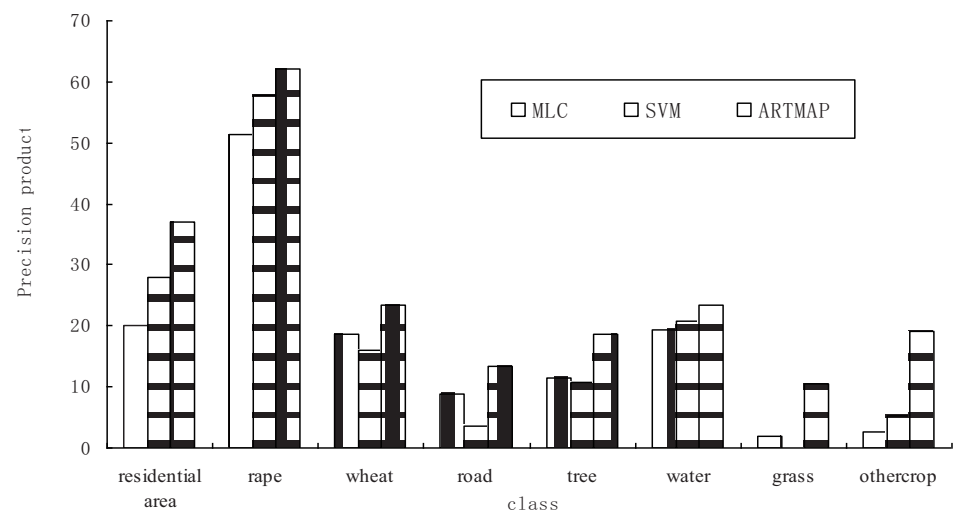

Fig. 2. Precision product of various classifiers

The non-parametric classifiers have better classification accuracy than the parametric classifiers. Non-parametric classifiers still have a low value of classification accuracy index. The ARTMAP has the highest overall 
accuracy, which was only $64.87 \%$; it may be influence by the mixture pixels (Ju et al., 2005). It is necessary to analysis the influence of the mixture pixels for the classification. Fig. 3 was showed the Classification results of various classifiers.

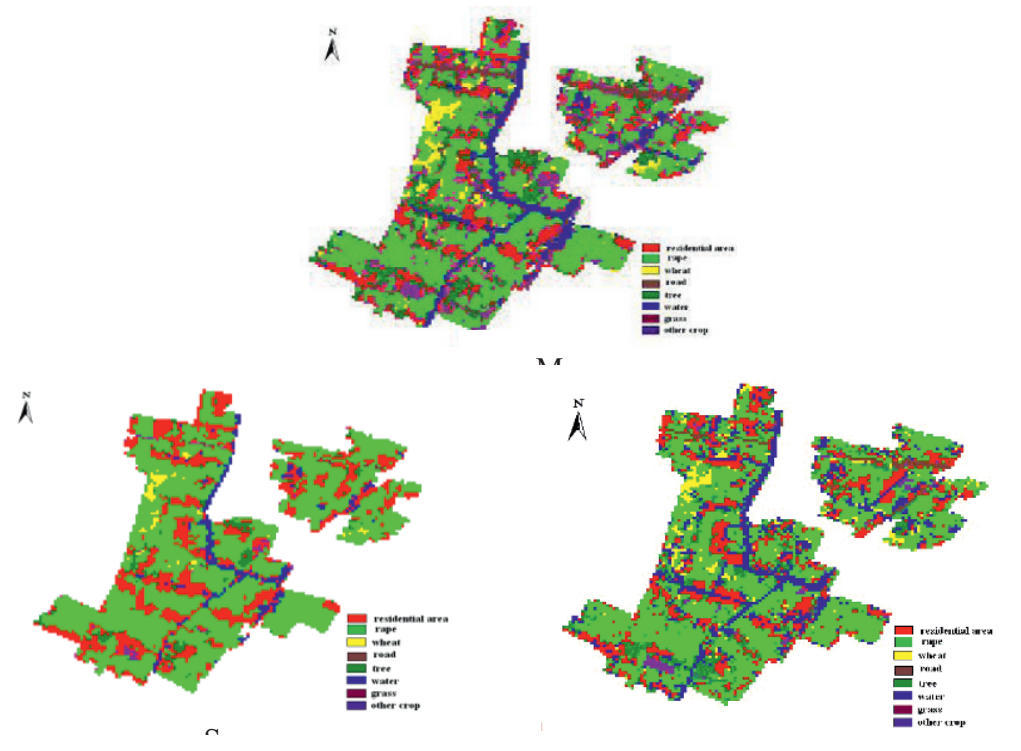

Fig.3. Classification results of various classifiers

\subsection{The influence of mixture pixels for Classification}

For the analysis of influence of mixture pixels, degraded the pixels of TM image to $90-100 \% 、 70-89 \%, 40-69 \%$ and $1-39 \%$, evaluated the classification accuracy of each grade. Fig. 4 showed the precision products of various classifiers for pixels of different pureness, and Fig. 5 showed Kappa coefficients and overall accuracy of various classifiers for pixels of different pureness. Based on the precision products, the higher the pixel pureness, the higher the precision products have, especially for the grade of $90-100 \%$. Furthermore, in the same pixel pureness grade, rape and water always had the higher precision products for each classifier. In general, the two non-parametric classifiers had higher Kappa coefficients and overall accuracy than the MLC, especially in the grade of $90-100 \%$, much more than $11-33 \%$ and $10-48 \%$ for overall accuracy and Kappa coefficients. From Fig. 4 and 5, it can be concluded that the ARTMAP was the much fine classify, especially in the grade of 90-100\%, Kappa coefficients and overall accuracy were nearly $100 \%$, and it's an excellent result. 

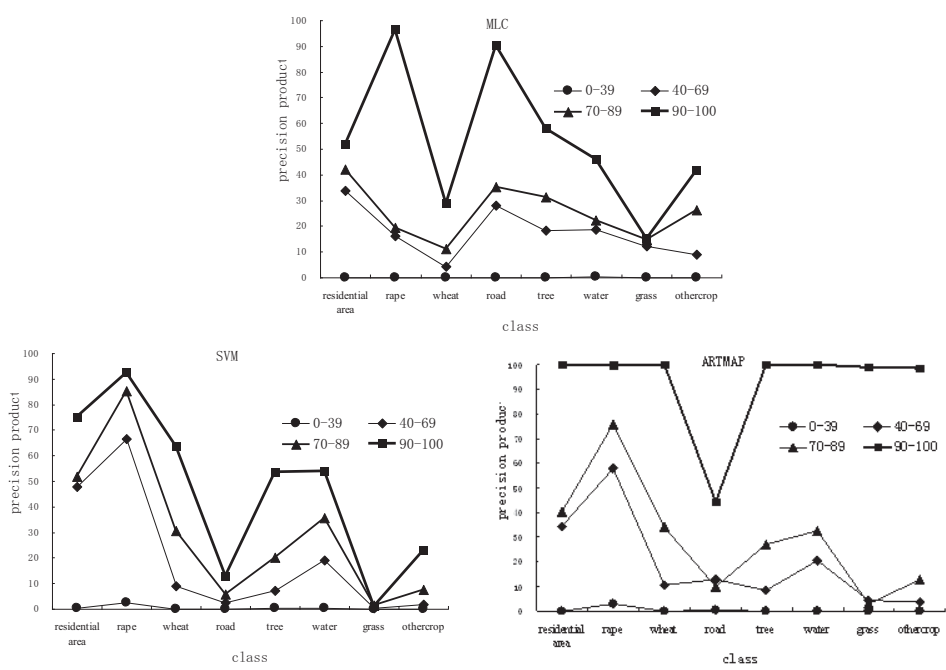

Fig. 4. Precision product of various classifiers for pixels of different
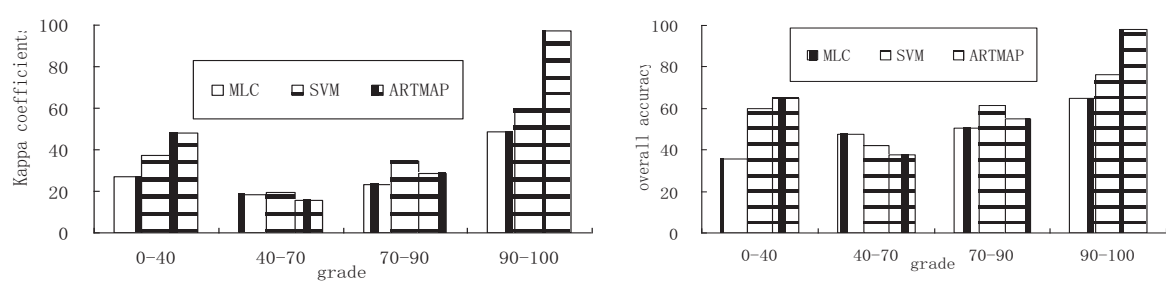

Fig. 5. Kappa coefficients and overall accuracy of various classifiers for pixels of different pureness

\section{CONCLUSIONS}

Supervised classification is a technique used commonly in the analysis of remotely sensed data. In the course of a classification many assumptions are made. To avoid problematic assumptions about the datasets made by conventional statistical classification algorithms, much attention has recently focused on non-parametric classifiers. This was illustrated for classifications with SVM and ARTMAP neural networks. For both types of non-parametric classifiers, the classification accuracy was prior to the parametric classifier-MLC, whereas the classification evaluation of both nonparametric classifiers were also not so much satisfaction.

There were a majority of mixture pixels of the TM image in the study area; it was also the representation for the agriculture region. The influence of the mixture pixels was the primary problem for the classification result and accuracy evaluation. The more fuzziness of the remote sensing data, the 
worse classification accuracy has. Only based on the spectral data and the pure pixel, the low classification accuracy result was reasonable. The ARTMAP classify represent much best than the rest of classify, especially for the grade of pure pixel (90-100\% pureness), Kappa coefficients and overall accuracy were nearly $100 \%$.

Overall, the results indicate that the non-parametric classifier may be a more preferable approach than the parametric classifier for some remote sensing applications and deserves further investigation. In the future study, fuzzy classification should be focused on as the important of the fuzziness influence for the classification accuracy. It may be not only appearing in the classification evaluation, but also in each step of the classification, such as the fuzzy train data and fuzzy classification result. In the next step, fuzzy classification may be a major study area we catch out.

\section{ACKNOWLEDGEMENTS}

Project supported by the National Natural Science Foundation of China (Nos. 40271078). We gratefully acknowledge the data providers including Junfeng Xv, La Chen, Qiuxiang Yi, Xiaohua Yang, of Institute of Agricultural Remote Sensing and Information Application, Huajiachi Campus, Zhejiang University, Hangzhou, China.

\section{REFERENCES}

C. C. Chang, C. J. Lin. LIBSVM : a library for support vector machines, 2001. Software available at http://www.csie.ntu.edu.tw/ cjlin/libsvm.

F. J. Cortijo. A Comparative Study of Classification Methods for Multispectral Images. PhD thesis, DECSAI, Universidad de Granada, Spain. 1995. Available at http://decsai.ugr.es/ cb/entrada_tesis.html, electronic edition.

M. J. Barnsley, S. L. Barr. Inferring urban land use from satellite sensor images using kernelbased spatial reclassi. Cation, Photogrammetric Engineering and Remote Sensing, 1996,62: 949-958.

N. Cristianini, J. Shawe-Taylor. An Introduction to Support Vector Machines. Cambridge University Press, Cambridge, UK, 2000.

R.O. Duda, P.E. Hart. Pattern Classification and Scene Analysis (New York: John Wiley \& Sons), 1973.

V. Vapnik. The Nature of Statistical Learning Theory. Springer-Verlag, New York. 1995. 